Original Paper

\title{
High Mobility Group B-1 (HMGB-1) Promotes Apoptosis of Macrophage- Derived Foam Cells by Inducing Endoplasmic Reticulum Stress
}

\author{
Han Wu Zheng Chen Jian-Zhou Chen Li-Gang Pei Jun Xie Zhong-Hai Wei \\ Li-Na Kang Lian Wang Biao Xu
}

Department of Cardiology, Drum Tower Hospital, Nanjing University Medical School, Nanjing, China

\section{Key Words}

High mobility group B-1 • Foam cell formation • Apoptosis • Endoplasmic reticulum stress

\begin{abstract}
Background/Aims: High mobility group B-1 (HMGB-1)-induced endoplasmic reticulum stress (ERS) has been implicated in inflammation and dendritic cell maturation. C/EBPhomologous protein (CHOP) is a vital component of ERS and apoptosis and plays a critical role in atherosclerosis. However, only a little information is available about the role of HMGB-1 in foam cell formation. Thus, the role of HMGB-1-induced ERS/CHOP pathway in apoptosis and formation of macrophage-derived foam cells is investigated. Methods: RAW264.7 cells were treated with oxidized low-density lipoprotein (oxLDL) in the absence and/or presence of HMGB-1, N-acetylcysteine (NAC, an antioxidant), glycyrrhizin (Gly, an HMGB-1 inhibitor), tunicamycin (TM, an ERS inducer), and 4-phenylbutyrate (4-PBA, an ERS inhibitor). Reactive oxygen species (ROS) production was examined by dihydroethidium (DHE) staining. Oil Red O staining, intracellular total cholesterol assay, and Dil-oxLDL uptake assay evaluated the accumulation of lipids in macrophages. Cell apoptosis was measured by flow cytometry and terminal deoxynucleotidyl transferase dUTP nick end labeling (TUNEL) staining. Western blot detected the expression of HMGB-1/ERS/CHOP pathway. Results: oxLDL induced HMGB1 translocation and secretion in a dose- and time-dependent manner, which was inhibited by NAC. oxLDL-induced lipid accumulation in macrophages was promoted synergistically by HMGB-1 that was attenuated by Gly. Moreover, TM synergized with oxLDL induced lipid accumulation and apoptosis of macrophages; however, 4-PBA alleviated the oxLDL-induced apoptotic foam cells. Additionally, the inhibition of ERS with 4-PBA suppressed the expression of HMGB-1-induced CHOP. Conclusions: OxLDL triggered HMGB-1 secretion in macrophages via oxidative stress. Furthermore, HMGB-1 promoted the formation and apoptosis of macrophage-derived foam cells via activation of ERS/CHOP pathway.

$\mathrm{H}$. Wu and Z. Chen contributed equally to this work.

\begin{tabular}{ll}
\hline Biao Xu & Department of Cardiology, Drum Tower Hospital, Nanjing University Medical School \\
& Nanjing 210008 (China) \\
& Tel. +8625831052 05, Fax +86 25-833-080-59, E-Mail submittingpaper@163.com
\end{tabular}
\end{abstract}

\section{KARGER}




\section{Introduction}

Atherosclerosis is the leading cause of deaths worldwide, with deteriorated clinical manifestations such as myocardial infarction, peripheral vascular diseases, and stroke [1]. Macrophages play a crucial role in all the stages of atherosclerosis development. Oxidized low-density lipoprotein (OxLDL), the strongest atherogenic factor, has been considered to promote the transformation of macrophages into foam cells, contributing to fatty streaks in atheroma. In addition, the apoptosis of foam cells has been recognized as a critical component in advanced plaques, which is the essential hallmark of vulnerable plaques [2-4] and leads to thrombosis-related acute ischemic cardiovascular events. However, the molecular mechanisms underlying apoptosis of macrophage-derived foam cells remain poorly understood.

High mobility group B-1 (HMGB-1), a conserved DNA-binding protein, is primarily expressed by macrophages. It can translocate from nucleus to cytosol and release into extracellular milieu under stress conditions. Extracellular HMGB-1 functions as a proinflammatory cytokine and plays a critical role in various pathological processes [5-7]. Early reports showed increased levels of serum HMGB-1 in patients with coronary artery disease [8, 9]. In apolipoprotein E-deficient (apoE ${ }^{-/}$) mice fed high-fat diet, treatment with neutralizing antibodies against HMGB-1 significantly prevented the development of atherosclerosis [10]. Further evidence revealed the critical role of HMGB-1 in human atherosclerosis lesions from the aorta, indicating high expression of HMGB-1 in carotid arteries [11], coronary arteries [12], and peripheral vessels [13]. In addition, macrophages have been considered to be primarily responsible for HMGB-1 expression in atherosclerosis [14], suggesting that the protein might promote macrophage-derived foam cells in plaques. However, the exact role of HMGB-1 in macrophage-derived foam cells remains unclear.

Endoplasmic reticulum stress (ERS), characterized by the accumulation of misfolded proteins in the ER lumen, exhibits homeostatic signaling pathways such as unfolded protein response (UPR). Accumulating evidence illustrated that severe ERS plays a critical role in foam cell formation and the development of atherosclerosis $[15,16]$. Furthermore, ERS induced C/EBP-homologous protein (CHOP)-related apoptotic signaling pathway in macrophages of advanced atherosclerotic plaques and promoted the rupture formation of plaques [2]. Thus, the present study aimed to investigate whether HMGB-1 induced the apoptosis of macrophage-derived foam cells via ERS/CHOP pathway.

\section{Materials and Methods}

\section{Cell culture and reagents}

RAW264.7 cells were obtained from Keygen Biotechnology(Nanjing, China). The cells were cultured in DMEM medium containing $10 \%$ fetal bovine serum at $37^{\circ} \mathrm{C}$ in a humidified incubator with $5 \% \mathrm{CO}_{2}$ until subconfluent. Then, the medium was replaced with serum-free media, and the cells treated for different experiments. OxLDL was obtained from Yiyuan Biotech (Guangzhou, China). HMGB-1 was from R\&D Systems (Minneapolis, MN, USA). N-acetylcysteine (NAC) was obtained from Beyotime Institute of Biotechnology (Haimen, China), glycyrrhizin (Gly) and 4-phenylbutyrate (4-PBA) from Sigma-Aldrich (St. Louis, MO, USA), and tunicamycin (TM) from Santa Cruz Biotechnology (Santa Cruz, CA, USA).

\section{Oil red 0 staining}

After the designated treatment, the cells were washed with cold phosphate-buffered saline (PBS) and fixed with $4 \%$ paraformaldehyde for $30 \mathrm{~min}$ at room temperature. Then, the cells were stained with $0.5 \%$ Oil Red 0 (Sigma-Aldrich, St. Louis, MO, USA) solution for $20 \mathrm{~min}$ at room temperature and counterstained with hematoxylin (Nanjing Jiancheng Bioengineering Institute, Nanjing, China) for 2 min, followed by PBS washes and microscopic enumeration. 


\section{Cellular Physiology Cell Physiol Biochem 2018;48:1019-1029 \\ \begin{tabular}{ll|l} 
and Biochemistry $10.1159 / 000491970$ & $\begin{array}{l}\text { D } 2018 \text { The Author(s). Published by S. Karger AG, Basel } \\
\text { www.karger.com/cpb }\end{array}$ \\
\hline
\end{tabular}}

Wu et al.: HMGB-1 in Apoptotic Foam Cell

\section{Uptake assay}

The cells were incubated with Dil-oxLDL for $24 \mathrm{~h}$, following which, the medium was removed. After two PBS washes, the cells were observed under fluorescence microscope (Olympus, Tokyo, Japan).

\section{Assessment of oxidative stress}

The macrophages were seeded in 6-well plates and subjected to serum starvation, followed by treatment as indicated in the Fig. 2. Then, intracellular oxidative stress was determined using dihydroethidium (DHE) according to the manufacturer's instructions. Briefly, the cells were incubated with $10 \mu \mathrm{mol} / \mathrm{L}$ DHE (Beyotime Institute of Biotechnology, Haimen, China) for $30 \mathrm{~min}$ at $37^{\circ} \mathrm{C}$ in the dark. Then, the cells were washed three times with PBS and observed under fluorescence microscope. Three fields were selected randomly in each well, and the DHE intensities quantified using the Image J software.

\section{Cell apoptosis assessment}

Terminal deoxynucleotidyl transferase dUTP nick end labeling (TUNEL) staining assay and annexin $\mathrm{V} /$ propidium iodide (PI) double staining assays were used to quantify cell apoptosis. For TUNEL staining, the cells were fixed with $4 \%$ paraformaldehyde for $30 \mathrm{~min}$ at room temperature, washed two times with PBS, and permeabilized with $0.1 \%$ Triton X-100 for $5 \mathrm{~min}$. Subsequently, the cells were subjected to TUNEL staining (Roche, Basel, Switzerland) for $1 \mathrm{~h}$ at room temperature in the dark, followed by 4,6-diamidino-2phenylindole (DAPI, Sigma-Aldrich, St. Louis, MO, USA) staining for the detection of cell nuclei. Images were acquired using a fluorescence microscope from three fields selected randomly in each well. The number of apoptotic cells was quantified using Image J software.

Annexin V/PI apoptosis detection kit was also used for examining cell apoptosis. Briefly, the cells were washed two times with cold PBS and resuspended in $500 \mu \mathrm{L}$ binding buffer. Subsequently, $5 \mu \mathrm{L}$ Annexin $\mathrm{V}$ and PI, each was added to the cells at room temperature in the dark. Finally, the cells were collected, resuspended in cold binding buffer for flow cytometry (Thermo Fisher Scientific, Waltham, MA, USA).

\section{Western blot}

Thenuclear and cytosolic proteins were extractedfrom the cellsusing the nuclear and cytoplasmic protein extraction kit (Beyotime Biotechnology, Haimen, China) according to the manufacturer's instructions. Total proteins were harvested in RIPA lysis buffer (Beyotime Biotechnology, Haimen, China) containing protease and phosphatase inhibitor tablets (Roche, Basel, Switzerland). The concentrations of the collected proteins were determined by bicinchoninic acid protein assay (BCA; Pierce, Rockford, IL, USA). Equivalent amounts of protein samples or cell supernatant were separated on 10-15\% sodium dodecyl sulfate polyacrylamide gel electrophoresis (SDS-PAGE) and transferred to polyvinylidene difluoride (PVDF) membranes (Merck Millipore, Billerica, MA, USA). After blocking with 5\% non-fat milk in tris-buffered saline containing $0.1 \%$ Tween 20 (TBST) for $1 \mathrm{~h}$ at room temperature, the membranes were probed with the following primary antibodies overnight at $4{ }^{\circ} \mathrm{C}$ : GAPDH (1:5000, Santa Cruz Biotechnology, Santa Cruz, CA, USA), Lamin B (1:2000, Santa Cruz Biotechnology, Santa Cruz, CA, USA), HMGB-1 (1:2000, Bioworld Technology Inc., St. Louis, MN, USA), Glucose regulated protein 78 (GRP78) (1:2000, Santa Cruz Biotechnology, Santa Cruz, CA, USA), phosphorylated inositol-requiring protein 1 (pIRE1) (1:1000, Cell Signaling Technology, Danvers, MA, USA), phosphorylated protein kinase RNA-like ER kinase (pPERK) (1: 1000, Cell Signaling Technology, Danvers, MA, USA), and CHOP (1: 2000, Cell Signaling Technology, Danvers, MA, USA). Subsequently, after three washes with TBST buffer, the membranes were incubated with horseradish peroxidase (HRP)conjugated secondary antibodies (1:10000, Santa Cruz Biotechnology) at room temperature for $2 \mathrm{~h}$. The immunoreactive bands were assessed using an enhanced chemiluminescence reagent (Pierce, Rockford, IL, USA). GAPDH and Lamin B were used as internal controls, and protein levels were quantified using Quantity One analysis software (Bio-Rad Laboratories Inc., Hercules, CA, USA).

\section{Immunofluorescence}

After the designated treatment, the cells were washed three times with cold PBS, fixed in $4 \%$ paraformaldehyde for $20 \mathrm{~min}$, and permeabilized with $0.1 \%$ Triton X-100 for $10 \mathrm{~min}$ to increase antigen accessibility. After blocking with 1\% BSA for $30 \mathrm{~min}$, the cells were reacted with a primary antibody against HMGB-1 (1:200, Bioworld Technology, Inc., St. Louis, MN, USA) overnight at $4{ }^{\circ} \mathrm{C}$. Subsequently, the cells were incubated with Alexa Fluor ${ }^{\circledR}$ 594-conjugated goat anti-rabbit secondary antibody (1:400, 
Invitrogen, Thermo Fisher Scientific, Inc., Waltham, MA, USA) for $1 \mathrm{~h}$ at $37^{\circ} \mathrm{C}$ in the dark. Then, the cells were counterstained with DAPI (Sigma-Aldrich, St. Louis, MO, USA) an examined under a fluorescence microscope (Olympus, Tokyo, Japan).

\section{Statistical analysis}

All experiments were conducted at least three times independently. The data were normalized to the respective control mean values and represented as means \pm SEM. Statistical analyses were performed using SPSS software (version 21.0; IBM SPSS, Armonk, NY, USA). ANOVA was used for multiple group comparison. Student-Neuman-Keels was used for post-hoc multiple comparisons. The statistical significance was set at 0.05 .

\section{Results}

OxLDL induced HMGB-1 translocation and secretion in macrophages in a dose- and timedependent manner

To determine the role of oxLDL on the translocation and secretion of HMGB-1 in macrophages, RAW264.7 cells were incubated with oxLDL at different concentrations ranging from $0-100 \mu \mathrm{g} / \mathrm{mL}$ for $24 \mathrm{~h}$ or $0,3,6,12$, and $24 \mathrm{~h}$. Western blot analysis revealed upregulation of HMGB-1 level in cell supernatant in a dose-dependent manner that reached a significant difference at $100 \mu \mathrm{g} / \mathrm{mL}$ (Fig. 1A, p<0.01). Also, we found that $100 \mu \mathrm{g} / \mathrm{mL}$ oxLDL induced the temporal profiles of HMGB-1 expression in cell supernatant (Fig. 1B). Thus, cells treated with $100 \mu \mathrm{g} / \mathrm{mL}$ oxLDL for $24 \mathrm{~h}$ were selected for the subsequent studies. The results shown in Fig. 1C indicated that oxLDL promoted the translocation of HMGB-1 from the nucleus to the cytoplasm, as evaluated by immunofluorescence.

\section{Oxidative stress contributed to oxLDL-induced HMGB-1 translocation in macrophages}

Oxidative stress was involved in HMGB-1 release in various cells. NAC, an antioxidant, was used to determine the role of oxidative stress in oxLDL-induced HMGB-1 translocation. As illustrated in Fig. 2A, oxLDL elevated the reduced nuclear HMGB-1 expression $(p<0.01)$, which was significantly suppressed by NAC $(p<0.05)$. Conversely, NAC partially but significantly attenuated the oxLDL-induced increased HMGB-1 expression in the cytoplasm $(p<0.05)$ (Fig. 2B). Furthermore, pretreatment with NAC normalized the elevated production of reactive oxygen species (ROS) induced by oxLDL in macrophages (Fig. 2C).

Fig. 1. Effect of oxLDL on HMGB-1 translocation and secretion in RAW264.7 cells. A, Effect of different concentrations of oxLDL on the HMGB-1 levels in cell supernatant. RAW264.7 cells were incubated with oxLDL for $24 \mathrm{~h}$ at different concentrations $(0,10,50$, $100 \mu \mathrm{g} / \mathrm{mL}$ ). B, Effect of $100 \mu \mathrm{g} / \mathrm{mL}$ oxLDL on HMGB1 secretion for different time points in RAW264.7 cells. RAW264.7 cells were stimulated with 100 $\mu \mathrm{g} / \mathrm{mL}$ oxLDL for $0,3,6,12,24 \mathrm{~h}$. C, Translocation of HMGB-1 in RAW264.7 cells induced by 100 $\mu \mathrm{g} / \mathrm{mL}$ oxLDL for $24 \mathrm{~h}$ (arrow indicates HMGB-1 localization). $\mathrm{N}$ (number of times the experiment was repeated) $=3$ in each group. NS, no difference between the two groups. ${ }^{* *} \mathrm{p}<0.01$ between the two groups. Scale bar $=100 \mu \mathrm{m}$.

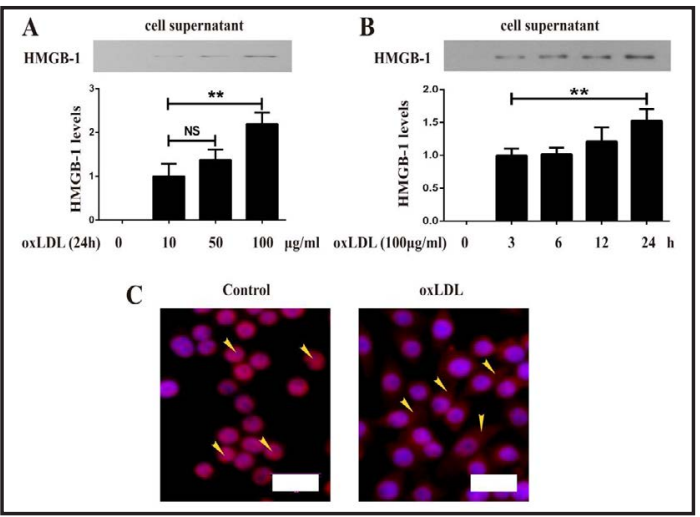


Fig. 2. Role of oxidative stress in oxLDL-induced HMGB-1 translocation in RAW264.7 cells. Cells were pretreated with $10 \mathrm{mmol} / \mathrm{L}$ NAC for $2 \mathrm{~h}$, followed by incubation with $100 \mu \mathrm{g} / \mathrm{mL}$ oxLDL for $24 \mathrm{~h}$. A, Expression of nuclear HMGB-1 in oxLDL-induced RAW264.7 cells with or without NAC treatment. B, Expression of cytoplasmic HMGB-1 in oxLDL-induced RAW264.7 cells with or without NAC treatment. C, Representative images of DHE staining in oxLDLinduced RAW264.7 cells with or without NAC treatment. NS, no difference between the two groups. ${ }^{*} \mathrm{p}<0.05$ and ${ }^{* *} \mathrm{p}<0.01$ between the two groups. The experiment was repeated three times in each group. Scale bar $=50 \mu \mathrm{m}$.

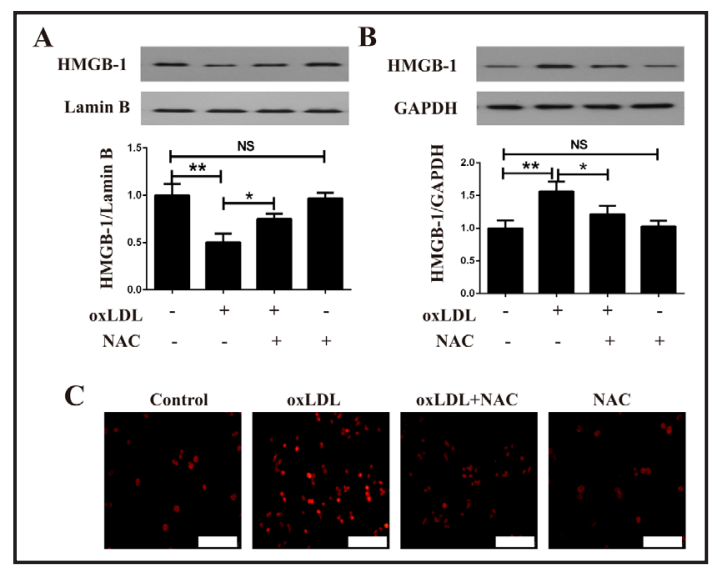

Fig. 3. Effect of HMGB-1 on foam cell formation in macrophages. After incubation with HMGB-1 (100 $\mu \mathrm{g} / \mathrm{mL})$ or Gly $(100 \mu \mathrm{mol} / \mathrm{L})$ for $2 \mathrm{~h}$, RAW264.7 cells were treated with oxLDL $(100 \mu \mathrm{g} / \mathrm{mL})$ or DiloxLDL $(50 \mu \mathrm{g} / \mathrm{mL})$ for $24 \mathrm{~h}$. A, Oil Red 0 staining in oxLDL-induced macrophages with HMGB-1 or Gly. Scale bar $=20 \mu \mathrm{m}$. B, The average IOD of lipid droplets stained using Oil Red 0 in oxLDL-induced macrophages with HMGB-1 or Gly. C, Quantitative analysis of intracellular total cholesterol content in oxLDL-induced macrophages with HMGB-1 or Gly. D, Fluorescence microscopy showed Dil-oxLDL uptake by RAW264.7 cells with HMGB-1 or Gly. Scale bar=50 $\mu \mathrm{m}$. The experiments were repeated three times in each group. ${ }^{*} \mathrm{p}<0.05$ and ${ }^{* *} \mathrm{p}<0.01$ between the two groups.

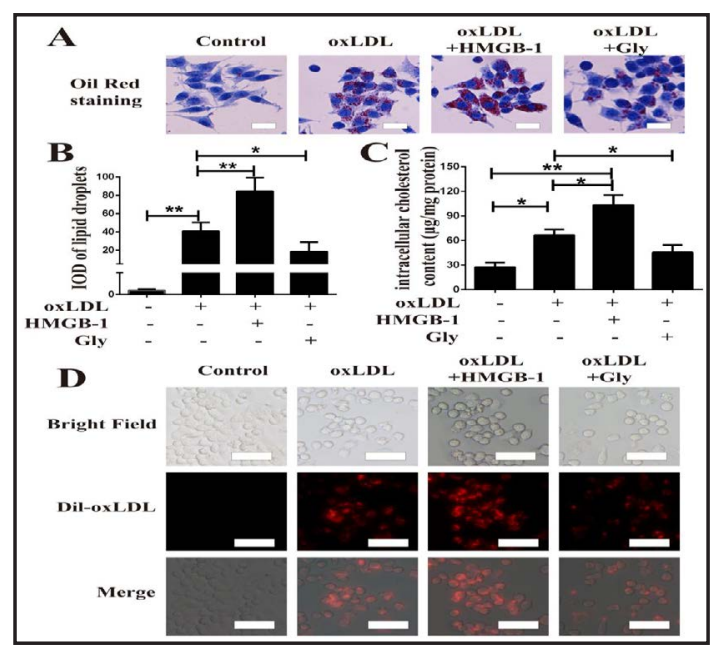

HMGB-1 promoted oxLDL-induced foam cell formation in macrophages

The present study examined the effect of HMGB-1 on macrophage-derived foam cells. As depicted in Fig. 3A, HMGB-1 facilitated the formation of foam cells induced by oxLDL in macrophages, as reflected by intense Oil Red 0 staining. To further substantiate the effect of HMGB-1 in oxLDL-induced foam cell formation, an inhibitor of HMGB-1 (Gly) was used in the subsequent experiments. Gly treatment significantly prevented the oxLDL-induced foam cells formation (Fig. $3 A$ and $B, p<0.05$ ). Similar results were obtained by intracellular total cholesterol assay, indicating that HMGB-1 promoted oxLDL-induced lipid content in RAW264.7 cells (Fig. 3C, p<0.05); however, Gly attenuated the level of lipid content in RAW264.7 cells induced by oxLDL (Fig. 3C, p<0.05). Furthermore, oxLDL-induced uptake and binding of Dil-oxLDL in RAW264.7 cells were enhanced by HMGB-1 but inhibited by Gly (Fig. 3D).

\section{HMGB-1 promoted oxLDL-induced apoptosis in macrophages}

To explain the effect of HMGB-1 on the apoptosis of oxLDL-treated macrophages, TUNEL staining (Fig. 4A and B) and Annexin/PI staining (Fig. 4C and D) were performed in this section. Both assays indicated that HMGB-1 boosted the apoptosis of macrophages induced by oxLDL $(\mathrm{p}<0.01$ and $\mathrm{p}<0.05$, respectively); however, pretreatment with Gly suppressed the oxLDL-induced apoptosis in macrophages (both $\mathrm{p}<0.05$ ). 
Fig. 4. Effect of HMGB-1 on oxLDL-induced apoptosis of RAW264.7 cells. Cells were treated as described in Fig. 3. A, Representative microphotographs of TUNEL staining in oxLDL-induced RAW264.7 cells with HMGB-1 or Gly. Nuclei were stained blue (DAPI) and green punctate staining represented TUNEL-positive cells; apoptotic cells were identified with DAPI and TUNEL double stained cells. B, Quantitative analysis of the percentage of TUNEL-positive cells relative to total oxLDL-induced RAW264.7 cells with HGMB-1 or Gly. C, The percentage of oxLDL-induced apoptotic cells with HGMB-1 or Gly by flow cytometry. D,

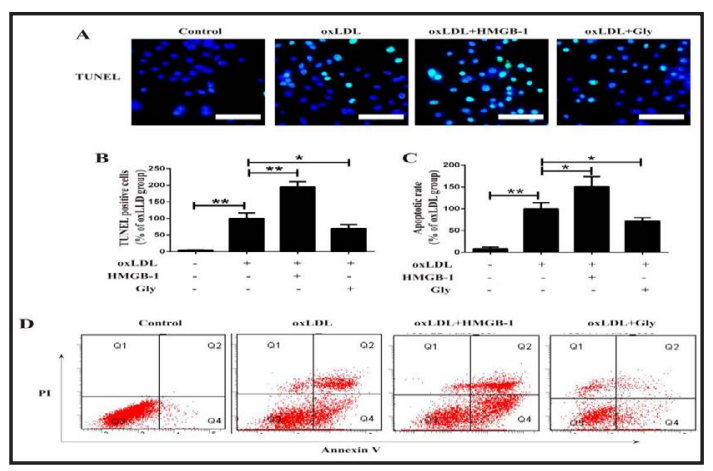
Representative histograms of Annexin/PI staining. The experiments were repeated three times in each group. ${ }^{*} \mathrm{p}<0.05$ and ${ }^{* *} \mathrm{p}<0.01$ between the two groups. Scale bar $=100 \mu \mathrm{m}$.

Fig. 5. Involvement of ERS in HMGB-1-induced apoptotic foam cells. RAW264.7 cells were pretreated with TM (4 $\mu \mathrm{g} / \mathrm{mL})$, HMGB-1 $(100 \mu \mathrm{g} / \mathrm{mL})$, or 4-PBA ( $5 \mathrm{mmol} / \mathrm{L}$ ) for $2 \mathrm{~h}$, followed by incubation with oxLDL $(100 \mu \mathrm{g} /$ $\mathrm{mL}$ ) for $24 \mathrm{~h}$. A, Oil Red 0 staining in oxLDL-induced macrophages with TM, HMGB-1, or 4-PBA. Scale bar $=50 \mu \mathrm{m}$. B, Average IOD of lipid droplets with Oil Red 0 staining in oxLDL-induced macrophages with TM, HMGB-1, or 4-PBA. C, Quantitative analysis of intracellular total cholesterol

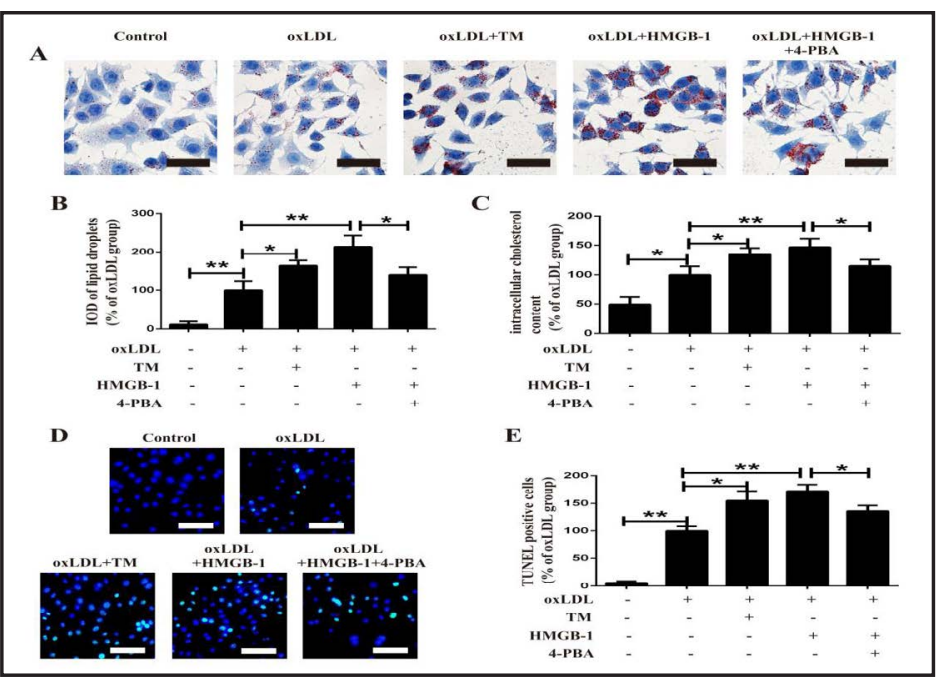
content in oxLDL-induced foam cells with TM, HMGB-1, or 4-PBA. D, Representative images of oxLDL-induced apoptotic cells with TM, HMGB-1, or 4-PBA. Nuclei were stained blue (DAPI) and green punctate represented TUNEL-positive cells; apoptotic cells were identified with DAPI and TUNEL double stained cells. Scale bar $=100 \mu \mathrm{m}$. E, Quantitative analysis of the percentage of TUNEL-positive cells relative to the total oxLDL-induced RAW264.7 cells with TM, HMGB-1, or 4-PBA. The experiments were repeated three times in each group. ${ }^{*} \mathrm{p}<0.05$ and ${ }^{* *} \mathrm{p}<0.01$ between the two groups.

\section{HMGB-1 promoted foam cell formation and apoptosis in oxLDL-induced macrophages via ERS}

Considering the ERS is a key modulator in the formation of foam cells, we determined whether HMGB-1 promoted apoptosis of foam cells in oxLDL-induced macrophages via ERS. Oil Red 0 staining (Fig. 5A and B) and intracellular total cholesterol quantitative assay (Fig. 5C) showed that the induction of ERS by tunicamycin (TM) exaggerated the oxLDL-induced foam cell formation as well as the level of HMGB-1; however, ERS inhibitor 4-phenylbutyrate (4-PBA) reversed the effect of HMGB-1 in macrophage-derived foam cells induced by oxLDL. As depicted in Fig. 5D, TM significantly promoted the apoptosis of oxLDL-treated macrophages $(\mathrm{p}<0.05)$, which was mimicked by the pretreatment with HMGB-1 $(\mathrm{p}<0.01)$. However, the stimulatory effect of HMGB-1 on apoptosis of macrophages was partially abolished by 4-PBA $(\mathrm{p}<0.05)$. 
Fig. 6. HMGB-1 induced ERS/CHOP signaling pathway in oxLDL-treated macrophages. RAW264.7 cells were stimulated with HMGB-1 $(100 \mu \mathrm{g} / \mathrm{mL})$ for $24 \mathrm{~h}$ after pre-incubation with 4-PBA ( $5 \mathrm{mmol} / \mathrm{L}$ ) for $2 \mathrm{~h}$. A, 4-PBA attenuated the expression of HMGB-1-induced GRP78 induced in RAW264.7 cells. B, 4-PBA attenuated the expression of HMGB-1-induced pPERK in RAW264.7 cells. C, 4-PBA did not exhibit any effect on HMGB-1induced pIRE1 expression in RAW264.7 cells. D, 4-PBA attenuated the expression of HMGB-1induced CHOP in RAW264.7 cells. The experiments were repeated three times in each group. ${ }^{*} \mathrm{p}<0.05$ and ${ }^{* *} \mathrm{p}<0.01$ between two groups.

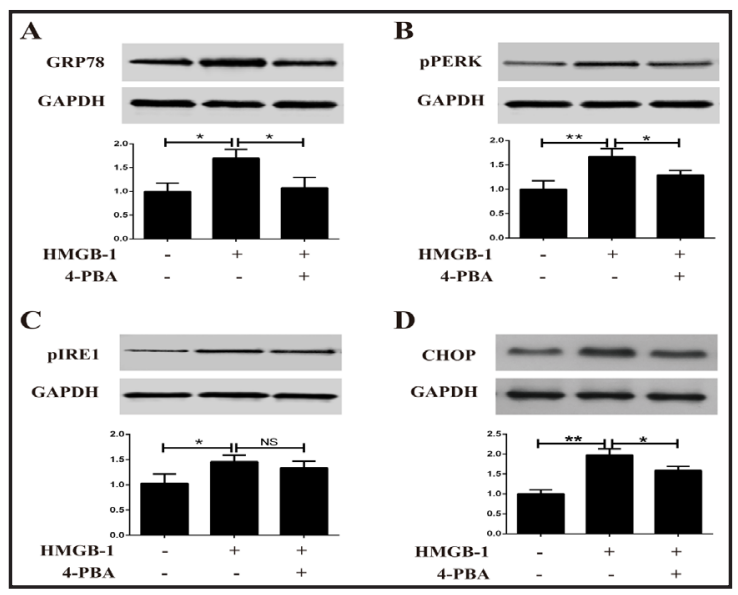

HMGB-1 upregulated CHOP expression via ERS in macrophages

Since ERS/CHOP pathway contributed to the apoptosis macrophages, we hypothesized that HMGB-1 regulated the CHOP expression via ERS in macrophages. To address this issue, we analyzed the expressions of ERS-related molecules, GRP78, pIRE1, pPERK, and CHOP. Western blot results (Fig. 6A-C) indicated that HMGB-1 promoted ERS in macrophages, as evident by the upregulated expression of GRP78 $(p<0.05)$, pIRE1 $(p<0.05)$, and pPERK $(p<0.01)$, and pretreatment with 4-PBA reversed the effect of HMGB-1 on GRP78 and pPERK protein levels. However, 4-PBA did not affect HMGB-1induced pIRE1 expression in RAW264.7 cells. Additionally, in response to the HMGB-1 challenge, RAW264.7 cells showed an increased expression of CHOP (Fig. 6D, p<0.01), which was normalized by 4-PBA (Fig. 6D, $\mathrm{p}<0.05$ ).

\section{Discussion}

The findings of the current study suggested that oxLDL upregulated the HMGB-1 translocation and secretion in macrophages. Additionally, HMGB-1 promoted the oxLDLinduced foam cell formation in macrophages via ERS. Also, HMGB-1-induced the apoptosis of macrophages via ERS/CHOP pathway. Therefore, these findings further demonstrated that ERS contributed to HMGB-1-induced apoptosis of macrophage-derived foam cells (Fig. 7).

HMGB-1, a ubiquitously expressed protein, is localized in the nucleus. It can be passively released by necrotic cells or actively secreted from active immune cells under specific conditions. Emerging evidence indicated that HMGB-1 could be released from macrophages by stimulators such as lipopolysaccharide [17] and inflammatory cytokines [14]. Interestingly, oxLDL induced HMGB-1 secretion in macrophages in a dose- and timedependent manner. These findings were consistent with those reported previously that cholesterol loading upregulated the HMGB-1 expression in vascular smooth muscle cells [18]; however, the underlying mechanisms are yet to be elucidated.

Accumulating evidence has indicated that oxidative stress is a primary modulator of HMGB-1 expression under pathological conditions. Our recent study showed that ROS genesis contributed towards the secretion of HMGB-1 from endothelial progenitor cells exposed to advanced glycation end production [19]. Concurrently, we also demonstrated that the inhibition of oxidative stress attenuated oxLDL-induced HMGB-1 translocation to cytoplasm and secretion, postulating that oxLDL promoted HMGB-1 secretion from macrophages via oxidative stress.

During the last decade, several studies revealed a correlation between HMGB-1 and atherosclerosis. Kalinina et al. demonstrated an increased expression of HMGB-1 in human atherosclerotic lesions [14], which was supported by subsequent studies. Another recent finding showed a local extension of HMGB-1 in atherosclerotic lesions of main cerebral and 
carotid arteries in humans [20]. In atherosclerosis animal models, HMGB-1 was increased in plaques from diabetic apoE deficiency mice [21]. Notably, the administration of anti-HMGB-1 neutralizing antibody suppressed the atherosclerosis by $55 \%$ in apoE-deficient mice [10]. Thus, HMGB1 may promote the in vivo development of atherosclerosis. Furthermore, macrophagederived foam cells and cell apoptosis were critical hallmarks in atherosclerotic lesions. Thus, the role of HMGB- 1 in oxLDL-induced foam cell formation was investigated. Consequently, we found that the treatment of HMGB-1 significantly promoted oxLDL-induced foam cell formation, as assessed by intense Oil Red 0 staining and intracellular cholesterol levels in

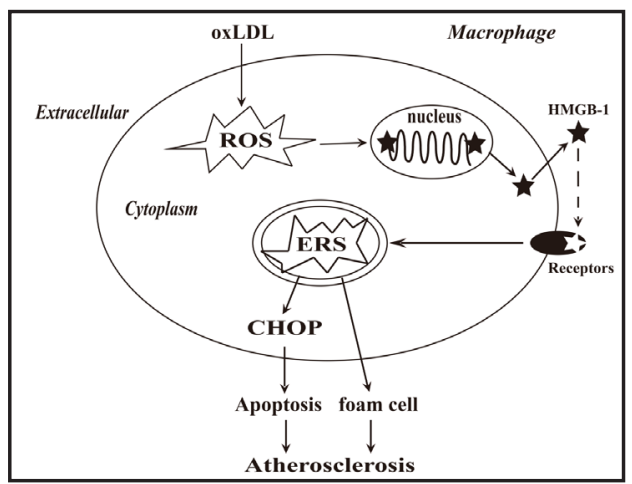

Fig. 7. Proposed mechanism, wherein HMGB-1 promoted oxLDL-induced apoptotic foam cells via ERS in macrophages. ROS, reactive oxygen species; ERS, endoplasmic reticulum stress. HMGB-1-treated RAW264.7 cells. Furthermore, pretreatment with Gly, an inhibitor of HMGB-1, reversed the effect of oxLDL on cholesterol uptake in macrophages, which confirmed the critical role of HMGB-1 in oxLDL-induced foam cell formation. Interestingly, macrophage apoptosis played a major role in vulnerable atherosclerotic plaques, a key characteristic of acute myocardial infarction [3,22]. Thus, in addition to its ability to promote foam cell formation, HMGB-1 has also been shown to facilitate apoptosis of oxLDL-treated RAW264.7 cells in the current study. These results were in agreement with those stated previously, wherein HMGB-1 induced the apoptosis of macrophages [23] and lymphocytes [24].

There is accumulating experimental evidence of the critical role of ERS in atherosclerosis development [25, 26] and foam cell formation [27-29]. In accordance to previous studies, our experiments showed that TM, an ERS inducer, promoted oxLDL-induced lipid accumulation and apoptosis of macrophages. Interestingly, it appears clearly from our experiments that the effects of ERS on foam cell formation are mimicked by the addition of HMGB-1. More recently, HMGB-1 has been shown to promote osteoblastic differentiation via ERS in aortic valve interstitial cells [30].Therefore, it is not completely understood but substantial data suggest that the proatherosclerotic effect of HMGB-1 on macrophages may be mediated via ERS. UPR is characterized by accumulation of unfolded proteins, mis-folded proteins and defective calcium homeostasis [31]. As an evolutionarily adaptive action, UPR is triggered to alleviate the disturbance of ER homeostasis. GRP78, a molecular chaperones, regulates protein folding and protein trafficking in ER lumen by binding to three stress sensors including PERK, IRE1 and activating transcription factor 6 (ATF6)[32]. However, GRP78 dissociates from the three proteins under stress conditions, leading to activation of the stress sensors, and eventually promotes initiation of ERS[33]. As shown previously, HMGB-1 induced the activation of ERS in endothelial cells in a dose-dependent manner, as reflected by the increased expression of PERK and IRE1 [34]. Thus, in the present study, exogenousexogenous HMGB-1 induced the expression of ERS markers (GRP78, pPERK, pIRE1) in macrophages. Additionally, HMGB1-induced expression of GRP78 and pPERK was reversed by 4-PBA (an inhibitor of ERS). Therefore, HMGB-1 might facilitate the oxLDL-induced lipid accumulation in macrophages via ERS.

Another interesting finding in the present study was that HMGB-1 promoted oxLDLinduced macrophage apoptosis that was significantly suppressed by 4-PBA. Accumulating evidence illustrated that sustained ERS could lead to the activation of apoptosis signaling pathway [27, 35]. These data provided additional evidence to the hypothesis that HMGB-1 may promote oxLDL-induced foam cell apoptosis via ERS. CHOP is a well-known apoptosisrelated signaling protein in macrophage-derived foam cells, contributing to the instability of atherosclerosis plaques $[2,36]$. In the current study, Western blot analysis showed that pretreatment with 4-PBA attenuated the HMGB-1-induced activation of ERS/CHOP signaling 
pathway, which was accompanied by a similar trend with the incidence of macrophage apoptosis. Taken together, these findings suggested that HMGB-1 facilitated oxLDL-induced apoptosis of macrophages via the ERS/CHOP pathway.

In conclusion, our results implied that HMGB-1 promoted oxLDL-induced macrophagederived foam cell apoptosis via ERS/CHOP pathway. Thus, drugs targeting HMGB-1 might be therapeutic strategies for preventing the development of atherosclerosis. However, the study presents some limitations. Herein, we investigated the effects of HMGB-1 on macrophages cell line; the data strength can be significantly improved by extrapolation into primary macrophages in future experiments. The HMGB-1 inhibitor was used in this study; nonetheless, additional genetic inhibition of HMGB-1 might improve the findings. Furthermore, additional investigations would provide the mechanism underlying the HMGB1-induced ERS in macrophages.

\section{Acknowledgments}

The current study was supported by grants from the Natural Science Foundation of China (Grants no. 81600285), Fundamental Research Funds for the Central Universities (Grants no. 021414380217), and Nanjing Medical Science and Technique Development Foundation (Grants no. qrx17114).

\section{Disclosure Statement}

The authors declare that they have no competing interests.

\section{References}

1 Weber C, Noels H: Atherosclerosis: current pathogenesis and therapeutic options. Nat Med 2011;17:14101422.

-2 Tsukano H, Gotoh T, Endo M, Miyata K, Tazume H, Kadomatsu T, Yano M, Iwawaki T, Kohno K, Araki K, Mizuta H, Oike Y: The endoplasmic reticulum stress-C/EBP homologous protein pathway-mediated apoptosis in macrophages contributes to the instability of atherosclerotic plaques. Arterioscler Thromb Vasc Biol 2010;30:1925-1932.

-3 Gonzalez L, Trigatti BL: Macrophage Apoptosis and Necrotic Core Development in Atherosclerosis: A Rapidly Advancing Field with Clinical Relevance to Imaging and Therapy. Can J Cardiol 2017;33:303-312.

4 Seimon T, Tabas I: Mechanisms and consequences of macrophage apoptosis in atherosclerosis. J Lipid Res 2009;50:S382-387.

5 Wu H, Chen Z, Xie J, Kang LN, Wang L, Xu B: High Mobility Group Box-1: A Missing Link between Diabetes and Its Complications. Mediators Inflamm 2016;2016:3896147.

-6 Ornellas FM, Ornellas DS, Martini SV, Castiglione RC, Ventura GM, Rocco PR, Gutfilen B, de Souza SA, Takiya CM, Morales MM: Bone Marrow-Derived Mononuclear Cell Therapy Accelerates Renal Ischemia-Reperfusion Injury Recovery by Modulating Inflammatory, Antioxidant and Apoptotic Related Molecules. Cell Physiol Biochem 2017;41:1736-1752.

7 Zhang J, Zhang J, Yu P, Chen M, Peng Q, Wang Z, Dong N: Remote Ischaemic Preconditioning and Sevoflurane Postconditioning Synergistically Protect Rats from Myocardial Injury Induced by Ischemia and Reperfusion Partly via Inhibition TLR4/MyD88/NF-kappaB Signaling Pathway. Cell Physiol Biochem 2017;41:22-32.

-8 Hashimoto T, Ishii J, Kitagawa F, Yamada S, Hattori K, Okumura M, Naruse H, Motoyama S, Matsui S, Tanaka I, Izawa H, Maruyama I, Nomura M, Ozaki Y: Circulating high-mobility group box 1 and cardiovascular mortality in unstable angina and non-ST-segment elevation myocardial infarction. Atherosclerosis 2012;221:490-495.

-9 Su Z, Lu H, Jiang H, Zhu H, Li Z, Zhang P, Ni P, Shen H, Xu W, Xu H: IFN-gamma-producing Th17 cells bias by HMGB1-T-bet/RUNX3 axis might contribute to progression of coronary artery atherosclerosis. Atherosclerosis 2015;243:421-428. 


\section{Cellular Physiology Cell Physiol Biochem 2018;48:1019-1029 \begin{tabular}{l|l} 
DOI: 10.1159/000491970 & O 2018 The Author(s). Published by S. Karger AG, Basel \\
www.karger.com/cpb
\end{tabular} \\ Wu et al.: HMGB-1 in Apoptotic Foam Cell}

10 Kanellakis P, Agrotis A, Kyaw TS, Koulis C, Ahrens I, Mori S, Takahashi HK, Liu K, Peter K, Nishibori M, Bobik A: High-mobility group box protein 1 neutralization reduces development of diet-induced atherosclerosis in apolipoprotein e-deficient mice. Arterioscler Thromb Vasc Biol 2011;31:313-319.

11 Moreno JA, Sastre C, Madrigal-Matute J, Munoz-Garcia B, Ortega L, Burkly LC, Egido J, Martin-Ventura JL, Blanco-Colio LM: HMGB1 expression and secretion are increased via TWEAK-Fn14 interaction in atherosclerotic plaques and cultured monocytes. Arterioscler Thromb Vasc Biol 2013;33:612-620.

12 Ahrens I, Chen YC, Topcic D, Bode M, Haenel D, Hagemeyer CE, Seeba H, Duerschmied D, Bassler N, JandeleitDahm KA, Sweet MJ, Agrotis A, Bobik A, Peter K: HMGB1 binds to activated platelets via the receptor for advanced glycation end products and is present in platelet rich human coronary artery thrombi. Thromb Haemost 2015;114:994-1003.

-13 Tsao CF, Huang WT, Liu TT, Wang PW, Liou CW, Lin TK, Hsieh CJ, Weng SW: Expression of high-mobility group box protein 1 in diabetic foot atherogenesis. Genet Mol Res 2015;14:4521-4531.

14 Kalinina N, Agrotis A, Antropova Y, DiVitto G, Kanellakis P, Kostolias G, Ilyinskaya O, Tararak E, Bobik A: Increased expression of the DNA-binding cytokine HMGB1 in human atherosclerotic lesions: role of activated macrophages and cytokines. Arterioscler Thromb Vasc Biol 2004;24:2320-2325.

-15 Tufanli O, Telkoparan Akillilar P, Acosta-Alvear D, Kocaturk B, Onat UI, Hamid SM, Cimen I, Walter P, Weber C, Erbay E: Targeting IRE1 with small molecules counteracts progression of atherosclerosis. Proc Natl Acad Sci U S A 2017;114:E1395-E1404.

-16 Clement M, Basatemur G, Masters L, Baker L, Bruneval P, Iwawaki T, Kneilling M, Yamasaki S, Goodall J, Mallat Z: Necrotic Cell Sensor Clec4e Promotes a Proatherogenic Macrophage Phenotype Through Activation of the Unfolded Protein Response. Circulation 2016;134:1039-1051.

17 Pisetsky DS: The expression of HMGB1 on microparticles released during cell activation and cell death in vitro and in vivo. Mol Med 2014;20:158-163.

-18 Porto A, Palumbo R, Pieroni M, Aprigliano G, Chiesa R, Sanvito F, Maseri A, Bianchi ME: Smooth muscle cells in human atherosclerotic plaques secrete and proliferate in response to high mobility group box 1 protein. FASEB J 2006;20:2565-2566.

19 Wu H, Li R, Wei ZH, Zhang XL, Chen JZ, Dai Q, Xie J, Xu B: Diabetes-Induced Oxidative Stress in Endothelial Progenitor Cells May Be Sustained by a Positive Feedback Loop Involving High Mobility Group Box-1 Oxid Med Cell Longev 2016;2016:1943918.

20 Umahara T, Uchihara T, Koyama S, Hashimoto T, Akimoto J, Haraoka J, Iwamoto T: Local extension of HMGB1 in atherosclerotic lesions of human main cerebral and carotid arteries. Histol Histopathol 2014;29:235-242.

21 Soro-Paavonen A, Watson AM, Li J, Paavonen K, Koitka A, Calkin AC, Barit D, Coughlan MT, Drew BG, Lancaster GI, Thomas M, Forbes JM, Nawroth PP, Bierhaus A, Cooper ME, Jandeleit-Dahm KA: Receptor for advanced glycation end products (RAGE) deficiency attenuates the development of atherosclerosis in diabetes. Diabetes 2008;57:2461-2469.

22 Linton MF, Babaev VR, Huang J, Linton EF, Tao H, Yancey PG: Macrophage Apoptosis and Efferocytosis in the Pathogenesis of Atherosclerosis. Circ J 2016;80:2259-2268.

23 Zhu XM, Yao YM, Liang HP, Liu F, Dong N, Yu Y, Sheng ZY: Effect of high mobility group box-1 protein on apoptosis of peritoneal macrophages. Arch Biochem Biophys 2009;492:54-61.

24 Wu ZS, Yao YM, Hong GL, Xu XP, Liu Y, Dong N, Zheng JY, Lu ZQ Zhao GJ, Zhu XM, Zhang QH, Sheng ZY: Role of mitofusin-2 in high mobility group box-1 protein-mediated apoptosis of T cells in vitro. Cell Physiol Biochem 2014;33:769-783.

-25 Ivanova EA, Orekhov AN: The Role of Endoplasmic Reticulum Stress and Unfolded Protein Response in Atherosclerosis. Int J Mol Sci 2016;17.

26 Tabas I: The role of endoplasmic reticulum stress in the progression of atherosclerosis. Circ Res 2010;107:839850.

27 Yao S, Tian H, Miao C, Zhang DW, Zhao L, Li Y, Yang N, Jiao P, Sang H, Guo S, Wang Y, Qin S: D4F alleviates macrophage-derived foam cell apoptosis by inhibiting CD36 expression and ER stress-CHOP pathway. J Lipid Res 2015;56:836-847.

28 Du H, Wang Y, Zhang Z, Yang J, Zhang J, Zhang Y: Fibronectin Overexpression Modulates Formation of Macrophage Foam Cells by Activating SREBP2 Involved in Endoplasmic Reticulum Stress. Cell Physiol Biochem 2015;36:1821-1834. 


\section{Cellular Physiology Cell Physiol Biochem 2018;48:1019-1029 \begin{tabular}{l|l|l} 
DOI: 10.1159/000491970 & $\begin{array}{l}\text { O 2018 The Author(s). Published by S. Karger AG, Basel } \\
\text { www.karger.com/cpb }\end{array}$ \\
\hline
\end{tabular} \\ Wu et al.: HMGB-1 in Apoptotic Foam Cell}

29 Yao S, Miao C, Tian H, Sang H, Yang N, Jiao P, Han J, Zong C, Qin S: Endoplasmic reticulum stress promotes macrophage-derived foam cell formation by up-regulating cluster of differentiation 36 (CD36) expression. J Biol Chem 2014;289:4032-4042.

30 Wang B, Cai Z, Liu B, Liu Z, Zhou X, Dong N, Li F: RAGE deficiency alleviates aortic valve calcification in ApoE/- mice via the inhibition of endoplasmic reticulum stress. Biochim Biophys Acta 2017;1863:781-792.

-31 Hotamisligil GS: Endoplasmic reticulum stress and atherosclerosis. Nat Med 2010;16:396-399.

-32 Zhang Y, Liu R, Ni M, Gill P, Lee AS: Cell surface relocalization of the endoplasmic reticulum chaperone and unfolded protein response regulator GRP78/BiP. J Biol Chem 2010;285:15065-15075.

33 Zhou AX, Tabas I: The UPR in atherosclerosis. Semin Immunopathol 2013;35:321-332.

-34 Luo Y, Li SJ, Yang J, Qiu YZ, Chen FP: HMGB1 induces an inflammatory response in endothelial cells via the RAGE-dependent endoplasmic reticulum stress pathway. Biochem Biophys Res Commun 2013;438:732738.

35 Scull CM, Tabas I: Mechanisms of ER stress-induced apoptosis in atherosclerosis. Arterioscler Thromb Vasc Biol 2011;31:2792-2797.

-36 Yao S, Tian H, Zhao L, Li J, Yang L, Yue F, Li Y, Jiao P, Yang N, Wang Y, Zhang X, Qin S: Oxidized high density lipoprotein induces macrophage apoptosis via toll-like receptor 4-dependent CHOP pathway. J Lipid Res 2017;58:164-177. 